\title{
HIGH TEMPERATURE BEHAVIOUR OF THE QCD COUPLING CONSTANT
}

\author{
R. BAIER ${ }^{\mathrm{a}}, \mathrm{B} \cdot \mathrm{PIRE}^{\mathrm{b}}$ and D. SCHIFF ${ }^{\mathrm{c}}$ \\ a Fakultät fur Physik, Universität Bielefeld, D-4800 Bielefeld, FRG \\ ' Centre de Physique Théorique ', Ecole Polytechnique, F-91128 Palaiseau, France \\ c Laboratoire de Physique Théorique des Hautes Energies ${ }^{2}$, Université Paris-Sud Orsay, F-91405 Orsay, France
}

Received 3 January 1990

\begin{abstract}
We investigate the finite temperature one loop renormalization of the pure QCD coupling constant in the real time formalism. The temperature dependence of the $\beta$ function is derived in a generalized momentum space subtraction scheme. Due to the occurrence of multiple Bose-Einstein distribution functions, we find $g^{2}(T) \approx T^{-3}$ for asymptotic temperatures at fixed momentum subtraction scale.
\end{abstract}

\section{Introduction}

The question of the high temperature behaviour of the QCD coupling constant is a crucial issue for improving perturbative calculations at finite $T$. It has often been taken for granted [1] that this $T$ behaviour is to be inferred from identifying the renormalization scale with $T$, thus leading to

$g^{2}(T) \approx \frac{1}{\ln \left(T / \Lambda_{\mathrm{QCD}}\right)}$.

This straightforward extension of the $T=0$ renormalization group (RG) results is not completely satisfactory. In ref. [2], Matsumoto et al. generalize the $R G$ approach at finite $T$ in the framework of the real time formalism [3]. In this framework, the ultraviolet divergences are the same as at $T=0$ but in addition to the freedom of choosing the usual momentum renormalization point, one has to deal with the arbitrariness of the temperature at which the renormalization parameters are determined. Within this finite temperature RG approach, the behaviour of the QCD coupling constant with respect to the temperature has been investigated by working out the solution of the one loop RG equations [4-7].

In this paper, we reanalyze this problem in a specific renormalization scheme and show that contributions have been overlooked which in fact dominate the high $T$ expansion of the QCD coupling constant. This scheme generalizes at finite $T$ the MOM renormalization procedure [8]. Propagators and vertices are renormalized at spacelike momenta and at a given temperature. We work in pure QCD and derive the one loop running coupling constant in the Feynman gauge in two MOM renormalization schemes attached to the three gluon vertex and the gluon-ghost vertex in the symmetric spacelike configuration for external lines $p^{2}=q^{2}=r^{2}=-M^{2}$ (fig. 1 ). We find that terms depending on the second and third power of the gluon (or ghost) Bose-Einstein statistical distribution are present ${ }^{\# 1}$ and dominate the asymptotic temperature behaviour of $g^{2}(T)$ at fixed $M$ :

$g^{2}(T) \approx(M / T)^{3}$.

1 Unité Propre du CNRS.

2 Unité Asscociée au CNRS.

\#1 In the collinear momentum configuration worked out in ref. [4], these terms do not contribute. 


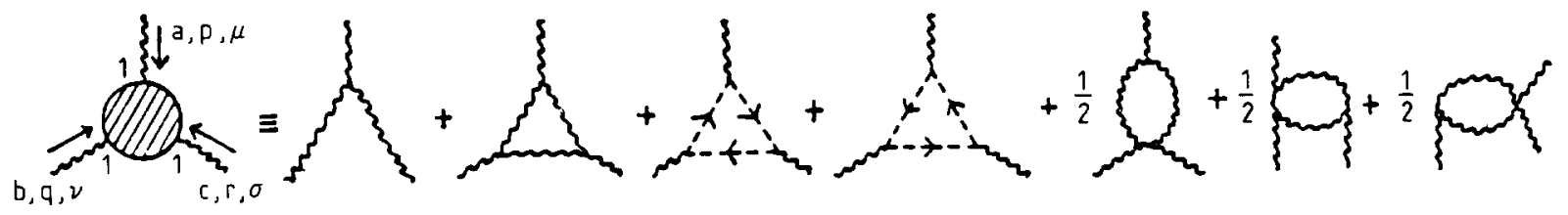

Fig. 1. The thermal (111) three gluon vertex up to one loop. The wavy (dotted) lines represent the gluon (ghost).

The above conventional inverse logarithmic behaviour is of course recovered in the special limit $T \rightarrow \infty, M / T$ fixed.

Another interesting result is the cancellation of logarithmic terms $(\ln M)$ in the renormalization constants when $T \rightarrow \infty$ at fixed $M$. Such terms have to be absent if the expected dimensional reduction of QCD at infinite temperature is realized [9]. In this respect, the $n_{\mathrm{B}}^{2}$ and $n_{\mathrm{B}}^{3}$ terms play an essential role.

The presence of contributions involving high powers of statistical distributions in Green's functions at the one loop level seems to be characteristic of the real time formalism ${ }^{\# 2}$. It may reopen the question of the comparison between this formalism and the imaginary time formalism [12].

Let us now outline the content of this paper. In section 2 , the three gluon vertex is calculated and the corresponding renormalization constant $Z_{1}$ is determined at a given temperature $T$. The corresponding running coupling constant is derived as a function of $T$ at fixed $M$. In section 3, the same procedure is applied to the gluonghost vertex. In section 4 , the high $T$ expansion of the running coupling constant is derived at fixed $M$. The expansion in $1 / T$ is given up to logarithmic terms and the $\ln M$ terms are shown to cancel.

\section{Three gluon vertex}

We start with the computation of the three gluon vertex renormalization constant at finite temperature. The one loop diagrams considered are shown in fig. 1 (neglecting contributions from the quark loop). They have the same structure as for $T=0$. In the real time formalism, however, there is a doubling of the fields (type- 1 and -2) [3]. We perform the momentum subtracted renormalization at finite $T$ for the real part of the (111) vertex, as indicated in fig. 1, following the prescriptions given in ref. [2]. Therefore only the free (11) propagators are needed, which are for the gluon field (in the Feynman gauge)

$\Delta_{\mu \nu}^{a b}(k)=\delta_{a b} g_{\mu \nu}\left(\frac{1}{k^{2}+\mathrm{i} \epsilon}-2 \pi \mathrm{i} \delta\left(k^{2}\right) n_{\mathrm{B}}\left(\left|k_{0}\right|\right)\right)$,

and for the ghost field

$\Delta_{\text {ghost }}^{a b}=-\delta_{a b}\left(\frac{1}{k^{2}+\mathrm{i} \epsilon}-2 \pi \mathrm{i} \delta\left(k^{2}\right) n_{\mathrm{B}}\left(\left|k_{0}\right|\right)\right)$,

where the indices $a, b$ denote the colour degrees of freedom $\# 3$. The Bose-Einstein distribution is

$n_{\mathrm{B}}\left(\left|k_{0}\right|\right)=\left[\exp \left(\beta\left|k_{0}\right|\right)-1\right]^{-1}, \quad \beta=1 / T$.

The following comment is in order. Inserting e.g. the gluon propagator (1) into the three point diagram with the gluon loop (fig. 1 ), $2^{3}=8$ terms result, which all contribute to the real part of the (111) vertex $\# 4$. One term contains the $T=0$ part, which was calculated by Celmaster and Gonsalves [8] in the renormalization scheme

\#2 In the imaginary time formalism at most a single power is present as stressed in refs. [10,11].

\#3 For the $T=0$ QCD Feynman rules we follow the conventions and notations of ref. [13].

\#4 Only four of them are taken into account in ref. [5]. 
under consideration. The other terms may be characterised by the powers of the Bose-Einstein distribution $n_{\mathrm{B}}$ : terms with one (considered in ref. [5]), but also with two and three powers in $n_{\mathrm{B}}$ are present. Performing the lengthy, but straightforward calculation for the diagrams in fig. 1 at the euclidean symmetric point $\left(p_{0}=q_{0}=r_{0}=0\right.$, $p^{2}=q^{2}=r^{2}=-M^{2}$ ), we get

$Z_{1}=Z_{1}^{T=0}-\frac{g^{2} N}{(4 \pi)^{2}}\left[H-\frac{3}{8} J_{1}^{(2)}-2 J_{3}^{(2)}-\frac{1}{4} J_{1}^{(3)}-\frac{4}{3} J_{3}^{(3)}\right]+\mathrm{O}\left(g^{4}\right)$,

where $Z_{1}^{T=0}$ is given in ref. [8] and need not be repeated here. The temperature dependence is contained in the functions $H$ and $J_{n}^{(m)}$, which are functions of $a \equiv \beta M$. The function $H$ contains the terms proportional to $n_{\mathrm{B}}$. Using the notation of ref. [5], it is given by $\# 5$

$H=-\frac{2}{3} F_{0}+\frac{7}{3} G_{0}-16 G_{2}$,

where e.g.

$$
\begin{aligned}
G_{0} & \equiv-4 \pi^{2} M^{2} \int \frac{\mathrm{d}^{4} k}{(2 \pi)^{3}} n_{\mathrm{B}}\left(\left|k_{0}\right|\right) \delta\left(k^{2}\right) \frac{1}{\left[(p+k)^{2}+\mathrm{i} \epsilon\right]\left[(k-q)^{2}+\mathrm{i} \epsilon\right]} \\
& =2 \mathrm{P} \int_{0}^{1} \mathrm{~d} y \int_{0}^{\infty} \frac{x \mathrm{~d} x}{\exp (\beta M x)-1} \frac{1}{x^{2}\left(y^{2}+3\right)-1} .
\end{aligned}
$$

The other functions $F_{0}, G_{2}$ are found in ref. [5]. The $n_{\mathrm{B}}^{2}$ and $n_{\mathrm{B}}^{3}$ terms are contained in the function $J_{n}^{(m)}$, i.e.

$J_{1}^{(m)} \equiv(4 \pi)^{2} M^{2} \int \frac{\mathrm{d}^{4} k}{2 \pi} n_{\mathrm{B}}^{m}\left(\left|k_{0}\right|\right) \delta\left(k^{2}\right) \delta\left((p+k)^{2}\right) \delta\left((k-q)^{2}\right)=\frac{8 \pi}{\sqrt{3}} \int_{0}^{\infty} \frac{\mathrm{d} x}{\sqrt{x^{2}+y^{2}}} \frac{1}{\left[\exp \left(\sqrt{x^{2}+y^{2}}\right)-1\right]^{m}}$

and

$J_{3}^{(m)}=\frac{8 \pi}{3 \sqrt{3} y^{2}} \int_{0}^{\infty} \frac{x^{2} \mathrm{~d} x}{\sqrt{x^{2}+y^{2}}} \frac{1}{\left[\exp \left(\sqrt{x^{2}+y^{2}}\right)-1\right]^{m}}$,

where $y^{2} \equiv \frac{1}{3} a^{2}$. These integrals are put into a form relevant for high temperature expansion as shown in ref. [14] in which the case $m=1$ was investigated.

In order to obtain the renormalized coupling constant $g_{\mathrm{R}}$ at finite $T$ in this renormalization scheme by

$g_{\mathrm{R}}=\frac{Z_{3}^{3 / 2}}{Z_{1}} g$,

we need the gluon-field renormalization constant, which is

$Z_{3}=Z_{3}^{T=0}-\frac{g^{2} N}{(4 \pi)^{2}}\left(\frac{4 \pi^{2}}{3 a^{2}}-3 F_{0}-F_{2}\right)+\mathrm{O}\left(g^{4}\right)$

as calculated in refs. $[5,8]$, renormalizing the gluon propagator at $p^{2}=-M^{2}$ and temperature $T$.

Applying the renormalization group equations discussed by Matsumoto et al. [2,5] with respect to the temperature $T$ and the arbitrary subtraction scale $M$, one obtains from eq. (9) the running coupling constant as a function of $T$ ( for fixed $M$ ),

$g_{\mathrm{R}}^{2}(T)=\frac{g_{\mathrm{R}}^{2}\left(T_{0}\right)}{1+\left[2 N /(4 \pi)^{2}\right] g_{\mathrm{R}}^{2}\left(T_{0}\right)\left[\Omega_{\text {glue }}(M / T)-\Omega_{\text {glue }}\left(M / T_{0}\right)\right]}$,

$\# 5$ We remark that we do not agree with ref. [5] on the coefficient of $F_{0}$. 
with

$\Omega_{\text {glue }}(a=M / T)=\frac{2 \pi^{2}}{a^{2}}-\frac{23}{6} F_{0}-\frac{3}{2} F_{2}-\frac{7}{3} G_{0}+16 G_{2}+\frac{3}{8} J_{1}^{(2)}+2 J_{3}^{(2)}+\frac{1}{4} J_{1}^{(3)}+\frac{4}{3} J_{3}^{(3)}$.

For fixed $M / T\left(=M_{0} / T_{0}\right)$ the solution reads

$g_{\mathrm{R}}^{2}(T)=\frac{g_{\mathrm{R}}^{2}\left(T_{0}\right)}{1+\left[g_{\mathrm{R}}^{2}\left(T_{0}\right) /(4 \pi)^{2}\right](22 / 3) N \ln T / T_{0}}$,

with the familiar logarithmic temperature dependence [1].

\section{The gluon-ghost vertex}

As for $T=0$ one may perform the momentum subtraction renormalization for the thermal (111) gluon-ghost vertex, instead of the three gluon vertex, i.e. instead of the definition, eq. (9), the renormalized coupling constant $g_{\mathrm{R}}$ is then expressed by [13]

$g_{\mathrm{R}} \equiv \frac{Z_{3}^{1 / 2} \tilde{Z}_{3}}{\tilde{Z}_{1}} g$

where $\tilde{Z}_{3}$ is the ghost wave function renormalization constant at finite $T$, given by ref. [5],

$\tilde{Z}_{3}=\tilde{Z}_{3}^{T=0}+\frac{g^{2} N}{(4 \pi)^{2}} F_{0}+\mathrm{O}\left(g^{4}\right)$

with $\tilde{Z}_{3}^{T=0}$ of ref. [8]. In contrast to the case of the three gluon vertex, the gluon-ghost renormalization constant $\tilde{Z}_{1}$ contains an ambiguity in the finite terms, which is due to the arbitrariness in the definition of the tensorial structure of this vertex at the one loop level [15]. Following ref. [15] it is expressed by introducing an additional parameter, denoted by $b$.

The result - in the Feynman gauge - is

$\tilde{Z}_{1}=\tilde{Z}_{1}^{T=0}(b)-\frac{g^{2} N}{(4 \pi)^{2}}\left[F_{0}+(1+2 b)\left(G_{0}+\frac{1}{8} J_{1}^{(2)}+\frac{1}{12} J_{1}^{(3)}\right)\right]+\mathrm{O}\left(g^{4}\right)$,

where $\tilde{Z}_{1}^{T=0}(b)$ is found in ref. [15]. The terms proportional to $J_{1}^{(m)}$ are due to $n_{\mathrm{B}}^{m}(m=2,3)$ contributions, which are not taken into account by Fujimoto et al. [5], who furthermore only discuss the case $b=0$.

The additional freedom in the parameter $b$ may be used to adjust it such that the Slavnov-Taylor identities of QCD [13], namely $Z_{1} / Z_{3}=\tilde{Z}_{1} / \tilde{Z}_{3}$, are satisfied in this renormalization scheme at a given finite $T$.

In a similar way to eqs. (11) and (12) the $T$ dependence of $g_{\mathrm{R}}(T)$ is obtained with

$\Omega_{\text {ghosi }}(a)=\frac{2 \pi^{2}}{3 a^{2}}-\frac{7}{2} F_{0}-\frac{1}{2} F_{2}-(1+2 b)\left(G_{0}+\frac{1}{8} J_{1}^{(2)}+\frac{1}{12} J_{1}^{(3)}\right)$

which is in general different from $\Omega_{\text {glue, }}$ eq. (12), showing the renormalization scheme dependence of $g_{\mathrm{R}}(T)$ at fixed $M$.

\section{High temperature behaviour}

Let us now examine the high temperature limit of the $\beta$ functions calculated in section 2 and 3 for the three 
gluon vertex and the gluon-ghost vertex respectively. We focus on the large $T$, fixed momentum $M$, region. The leading behaviour is obtained from the small $a$ behaviour of the $J_{n}^{(m)}(a)$ functions defined above in eqs. (7), (8). Since $n_{\mathrm{B}}(x) \approx x^{-1}$ for small $x$, one gets for $m \geqslant n$

$J_{n}^{(m)}(y) \approx y^{1-n} \int_{0}^{\infty} \mathrm{d} x x^{n-1}\left(x^{2}+y^{2}\right)^{-(1+m) / 2}$

which by rescaling leads to

$J_{n}^{(m)}(y) \approx y^{-m}$

for small $y$. The leading behaviour of $\Omega_{\text {glue }}(a)$ is thus $a^{-3}$, i.e. the running coupling constant behaves as

$g_{\mathrm{R}}^{2}(T) \approx M^{3} / T^{3}$

for large $T / M$. Let us now discuss the high $T$ expansion of $\Omega_{\text {glue }}$ and $\Omega_{\text {ghost }}$ up to logarithmic terms. The asymptotic expansions of the $F_{0,2}$ and $G_{0.2}$ functions are given in ref. [5]. Using Haber and Weldon's techniques [14], we get the corresponding expansion for the $J_{n}^{(m)}$ functions. They are

$J_{1}^{(2)}=\frac{8 \pi}{\sqrt{3}}\left(\frac{3}{a^{2}}-\frac{\sqrt{3} \pi}{2 a}-\frac{5}{12} \ln a+\ldots\right)$,

$J_{3}^{(2)}=\frac{8 \pi}{\sqrt{3}}\left(-\frac{\ln (a / 2 \sqrt{3})}{a^{2}}-\frac{\pi^{2}}{6 a^{2}}+\frac{\pi}{2 \sqrt{3} a}+\frac{5}{72} \ln a+\ldots\right)$,

$J_{1}^{(3)}=\frac{8 \pi}{\sqrt{3}}\left(\frac{\pi 3 \sqrt{3}}{4 a^{3}}-\frac{9}{2 a^{2}}+\frac{\pi \sqrt{3}}{2 a}+\frac{3}{8} \ln a+\ldots\right)$,

$J_{\xi}^{(3)}=\frac{8 \pi}{\sqrt{3}}\left[\frac{\pi \sqrt{3}}{4 a^{3}}+\frac{3}{2 a^{2}} \ln \left(\frac{a}{2 \sqrt{3}}\right)+\frac{2 \pi^{2}-3}{12 a^{2}}-\frac{\pi}{2 \sqrt{3} a}-\frac{1}{16} \ln a+\ldots\right]$.

From this, we obtain

$\Omega_{\text {glue }}=\frac{25 \pi^{2}}{6 a^{3}}+\mathrm{O}\left(\frac{1}{a^{2}}\right)$

and

$\Omega_{\text {ghost }}=-\frac{1+2 b}{2} \frac{\pi^{2}}{a^{3}}+\mathrm{O}\left(\frac{1}{a^{2}}\right)$.

They are equal for the special value of the ghost parameter $b=-\frac{14}{3}$ and therefore yield the same running coupling constant at large $T$.

We finally comment on the terms proportional to $\ln M / T$ in the high $T$ expansion of the renormalization constants. The $T=0$ parts contain terms $\ln M / \mu$, where $\mu$ is the scale in the applied dimensional regularization procedure. As is already known form ref. [16] these terms combine with $\ln M / T$ to yield a $\ln T / \mu$ dependence in the wave function renormalization constants $Z_{3}$ and $\tilde{Z}_{3}$, respectively. With the help of the expansions, eq. (21), we also find it true for the vertex renormalization constants $Z_{1}, \tilde{Z}_{1}$. However, we stress that for this cancellation of the $\ln M$ dependence, the terms proportional to $n_{\mathrm{B}}^{2}$ and $n_{\mathrm{B}}^{3}$ are crucial.

To summarize, the thermal $\beta$ function, $\beta_{T}=T(\mathrm{~d} / \mathrm{d} T) g_{\mathrm{R}}^{2}(T, M) / 4 \pi^{2}$, behaves for large $T$ and in the investigated renormalization scheme (for the three gluon vertex) as

$\beta_{T}=-\frac{25 \pi^{2} N}{4}\left(\frac{T}{M}\right)^{3} \frac{g_{\mathrm{R}}^{2}}{4 \pi^{2}}+\mathrm{O}\left(\frac{T^{2}}{M^{2}}\right)$. 
As a consequence of the $\ln M$ term cancellation, the constant term in this expansion is exactly the same as for the usual $T=0 \beta$ function: $-\frac{11}{6} N\left(g_{\mathrm{R}}^{2} / 4 \pi^{2}\right)^{2}$.

\section{Acknowledgement}

Partial support of this work by "Projets de Coopération et d'Echange" (PROCOPE) is gratefully acknowledged.

\section{Note added in proof}

After completion of this work, we received a paper by Nakkagawa, Niegawa and Yokota [17] where similar considerations are developed.

\section{References}

[1] M.B. Kisslinger and P.D. Morley, Phys. Rep. 51 (1979) 63; J.C. Collins and M.I. Perry, Phys. Rev. Lett. 34 (1975) 1353.

[2] H. Matsumoto, I. Ojima and H. Umezawa, Ann. Phys. (NY) 152 (1984) 348;

H. Matsumoto, Y. Nakano and H. Umezawa, Phys. Rev. D 29 (1984) 1116.

[3] For a review see N.P. Landsman and Ch.G. van Weert, Phys. Rep. 145 (1987) 141.

[4] H. Nakkagawa, A. Niegawa and H. Yokota, Phys. Rev. D 38 (1988) 2566.

[5] Y. Fujimoto and H. Yamada, Phys. Lett. B 195 (1987) 231; B 200 (1988) 167; INS preprint Rep 642 (1987), unpublished.

[6] K. Enqvist and K. Kajantie, Mod. Phys. Lett. A 2 (1987) 479.

[7] N.P. Landsman, Phys. Lett. B 232 (1989) 240.

[8] W. Celmaster and R. Gonsalves, Phys. Rev. D 20 (1979) 1420.

[9] R. Alvarez-Estrada, Ann. Phys. (NY) 174 (1987) 442; Fortschr. Phys. 36 (1988) 145;

N.P. Landsman, Nucl. Phys. B 322 (1989) 498;

E.L. Koopman, Dimensional reduction at finite temperature, thesis, University of Amsterdam (1988), unpublished.

[10] E. Braaten and R.D. Pisarski, Fermilab report PUB-89/152T (1989).

[ 11 ] J. Frenkel and J.C. Taylor, University of Cambridge preprint DAMPTP89-23 (1989).

[12] Y. Fujimoto, R. Grigjanis and H. Nishino, Phys. Lett. B 141 (1984) 83;

Y. Fujimoto and R. Grigjanis, Z. Phys. C 28 (1985) 395;

Y. Fujimoto and H. Yamada, Z. Phys. C 37 (1988) 265.

[13] T. Muta, Foundations of quantum chromodynamics (World Scientific, Singapore, 1987).

[14] H.E. Haber and H.A. Weldon, J. Math. Phys. 23 (1982) 1852.

[15] P. Pascual and R. Tarrach, Nucl. Phys. B 174 (1980) 123.

[16] H.A. Weldon, Phys. Rev. D 26 (1982) 1394.

[17] H. Nakkagawa, A. Niegawa and H. Yokota, Nara University report NUTP/89-2. 